\title{
Design of Digital Barometer Based on Single-Chip Microcomputer Control
}

\author{
Weisheng Zheng and Yimei Liu \\ Jiangxi Technical College of Manufacturing, Department of Information Engineering
}

Keywords: Digital barometer; Single-chip machine; Sensor; Hardware realization method

\begin{abstract}
The core component of barometer is the pressure sensor, which plays a very important role in monitoring pressure, controlling pressure changes and measuring other physical quantities. Barometer obtains the air pressure data through changes of air pressure at different heights. According to the meteorological regulations, the weight of air column effecting each unit of area is called air pressure. Digital barometer is widely applied to mining enterprises, electronic products and outdoor operation, and after a long period of development, it is becoming increasingly accurate with more diversified functions, and is gradually developing towards being intelligent and multi-functional. This paper introduces the digital barometer based on single-chip control in details, analyzes the overall structure of barometer and introduces the way to realize both software and hardware of barometer as well as adjust and simulate the digital barometer system to guarantee the reliability and stability of the digital barometer system's functions.
\end{abstract}

\section{Introduction}

Digital barometer has been widely applied in various engineering environment: mining fields, weather station, environmental protection and labs, etc. It is an equipment directly changing the measure air pressure into current or voltage signal that can be easily transmitted and detected using the pressure-sensitive components, and then real-timely display them through the subsequent circuit processing. Its core component is barometer, which plays a vital role in controlling pressure changes, monitoring pressure and measuring physical parameters. Single-chip machine has some advantages, such as convenient, simple and flexible controlling.

At present, barometer with single pressure-measuring function has default setting value that cannot be adjusted or revised, so it is not convenient to use them. This paper has made improvement to design in various aspects to develop a multi-functional digital barometer, which can modify the set value if necessary and input it to the system with huge range of work temperature and measured air pressure as well as high accuracy, stability and strong ability to anti-interference. Its multi-display function can allow users to intuitively monitor the current status of each signal (such as showing temperature, supply voltage and pressure voltage, etc.). The serial communication port of the system can transmit the measured data to the computer for various processing and make the barometer intelligent, bringing great convenience to users.

\section{Hardware Realization of Digital Barometer}

Various factors need to be taken into consideration in constructing hardware of digital barometer, such as its stability, complexity, manufacturing costs and maintenance costs, etc. This chapter introduces the composition of digital barometers' hardware equipment to establish a set of stable and systematic hardware equipment.

Air Pressure Sensor. Air pressure sensor exists at the core position of the entire air pressure structure and to choose an appropriate air pressure sensor, various factors need to be taken into consideration: measuring accuracy, measuring range, temperature changes, and absolute air pressure value, etc. Digital barometer is to measure the absolute air pressure value, so it is necessary to have air pressure sensor that can measure the absolute air pressure value. In order to simplify the circuit design and improve barometer's stability and anti-interference capacity, barometer also needs to have temperature compensation. According to the above requirements, choose the 
MAX41000A type of Motorda barometer in this digital air pressure sensor. Temperature compensation range of this air pressure sensor is $-40 \sim 125^{\circ} \mathrm{C}$, and the pressure-bearing range is $20 \sim 1050 \mathrm{kPa}$. The air pressure sensor has good linearity within this pressure range and the range of voltage output signal is $0.3 \sim 4.65 \mathrm{~V}$. Air pressure sensor can be used to measure the absolute air pressure, and its specific output relationship is as follows:

$V_{\text {out }}=V_{s}(0.01059 P-0.1528) \pm$ Error

In the formula, $V_{s}$ is the work voltage, $\mathrm{P}$ is the air pressure value, and $\mathrm{V}$ is the output voltage.

V/F Transformation. Air pressure sensor measures the current and voltage signal of the measured air pressure, but it cannot be recognized by single chip machine, so V/F component needs to transfer the input voltage value into pulse string that can be recognized by single chip machine. Although the V/F component itself is not a quantizer, the existence of timer and counter can realize the conversion of $A / D$ to convert the simulated voltage signal into pulse string with strong anti-interference ability, capable of realizing distance transmission and being input into computer.

LM331 can use the single and double power supply and allowed range of voltage is $5 \sim 40 \mathrm{~V}$ and the maximum non-linear error is $0.001 \%$. The LM331 pressure frequency conversion relationship is:

$\begin{aligned} f_{0} & =K V_{i} \\ \text { Herein, } K & =R_{0} /\left(2.09 R_{i} C_{i} R_{L}\right), R_{s}=R_{s 1}+R_{s 0}\end{aligned}$

\section{System's Framework Structure and Overall Design of System Hardware}

The whole system is through the pressure sensor to sense the atmospheric pressure, and the measured pressure conversion is an analog voltage signal, due to the voltage signal is weak, only a few tens of MV, so through the integrated amplifier LM324 will signal amplification, and then is connected with the MCU ADC port for A / D conversion. The linear relationship between voltage and pressure, can determine the change of air pressure value, plus pressure reference value can be obtained pressure value. But this value is not accurate, because the atmospheric pressure is also affected by the subtle influence of temperature, pressure and temperature compensation must be added to get the exact value of the pressure value. The temperature compensation value is the current temperature through the temperature sensor, the temperature and pressure relation determined. Finally, under the control of the MCU through eight 4 segment digital tube display the corresponding pressure value, temperature value, voltage value, etc.

The overall design is based on the function analysis to design the system's structure framework, development procedure and development principle. Fig. 1 is the structure framework of digital barometer system. 


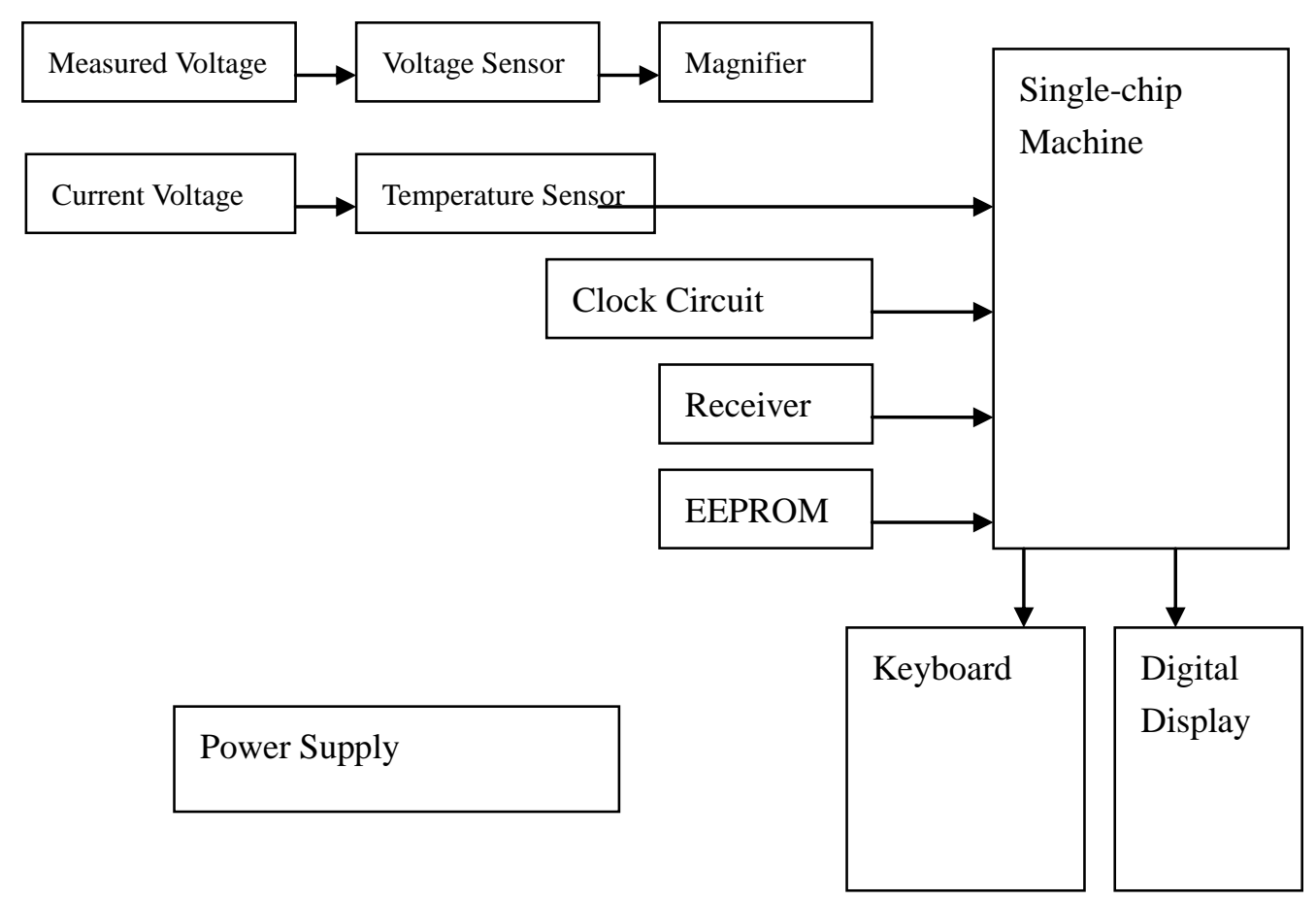

Figure 1. System Framework

(1) Air pressure sensor (MPX100) and temperature sensor (DS18b20cn)

Air pressure sensor senses the air pressure and converts it into voltage signal; temperature sensor serves to convert the current temperature into voltage signal.

(2) Magnifier (LM324)

Magnifier magnifies the voltage signal converted by sensor to facilitate the single-chip machine in signal processing.

(3) Clock Circuit

It records current time.

(4) Telecommunication circuit

The telecommunication between the $I^{2} C$ line and upper machine makes it convenient to timely process the obtained voltage value.

(5) Memory Expansion Circuit

EEPROM expands so that the entire system can be carried out in large quantities of data stored in the processing. System hardware system is divided into two parts: the core module and the peripheral expansion module. The core modules include AVR microprocessor, memory, oscillator, reset, bus interface. Peripheral expansion module includes power supply, keyboard, LED display, etc.

\section{Conclusion}

Intelligent digital barometer has broad application prospect, and the current barometer design is not so comprehensive, so it cannot satisfy the requirement of being timely and small. This paper mainly deals with the following aspects:

(1) It firstly introduce the significance of studying intelligent digital barometer based on AVR and the current development both in China and abroad as well as the development based on collecting and searching a large number of domestic and foreign literatures.

(2) It analyzes the functions of digital barometer system and conducts the overall design, including the necessity to adopt AVR, design of the system framework, development process and development principles. Thus, it has universal significance to designing digital barometer system based on AVR. 
(3) It analyzes the hardware design of digital barometer based on AVR, including data collection, data processing and data displayed circuit.

\section{References}

[1] Pike J M, Bargen D W. The NCAR Digital Barometer.[J]. Bulletin of the American Meteorological Society, 1976, 57(57):1106-1112.

[2] Wang, Gui You, G. U. Zhao, and B. Zang. "Test of Certain Digital Barometer." Chinese Medical Equipment Journal (2010).

[3] Pascoe, R. W., and J. M. Rüeger. "Testing a precise hand-held digital barometer." Australian Surveyor 34.34(1989):502-513.

[4] Gao, Shan Qing, and X. L. Wei. "The Analysis of Digital Barometer Processing Algorithms." Journal of Sichuan Ordnance (2013).

[5] Shen S X, Ai-Hua H U. The Design and Implementation of Digital Barometer Base on Single Chip Microcomputer [J]. International Electronic Elements, 2004.

[6] Hu, Fan, and Z. Y. Chen. "The principle and application of PTB220 series digital barometers." Meteorological Hydrological \& Marine Instruments (2008).

[7] Phys, Mueller Alfred Dipl. "Mercury barometer with digital read=out - has column height measured ultrasonically with microprocessor control and compensation.", DE2650227. 1978.

[8] Nief G, Millier P, Brebion N, et al. New portable digital microbarometer DMB one[C]// EGU General Assembly Conference Abstracts. EGU General Assembly Conference Abstracts, 2012.

[9] Alexandru P D, Irina M, Alice C. Consumers`Attitude towards Consumer Protection in the Digital Single Market, as Reflected by European Barometers[J]. Amfiteatru Economic Journal, 2014, 16(36):563.

[10]Ridleysiegert T. Customer acquisition barometer 2015[J]. Journal of Direct Data \& Digital Marketing Practice, 2015, 17(2):93-100.

[11]Jain, Yashraj, and A. Colwell. "DMA Customer Acquisition Barometer 2014." Journal of Direct Data \& Digital Marketing Practice 15.4(2014).

[12] Vanlonden A. The evaluation of automatic barometers, illustrated by the Nakaasa barometer [J]. Nasa Sti/recon Technical Report N, 1987, 88. 